\title{
Study of A Bus Voltage Control Strategy for Two-Way AC/DC Current Converter in DC Microgrid
}

\author{
Ningkang Zheng ${ }^{1 *}$, Xiangyang Yan ${ }^{1}$, Huanruo Qi ${ }^{1}$, Yilong Kang ${ }^{1}$ \\ ${ }^{1}$ State Grid Henan Economic Research Institute, Zhengzhou, 450000, China
}

\begin{abstract}
In an isolated DC microgrid consisting of photovoltaic cells, an energy storage unit and load, reasonable control of grid-connected two-way AC/DC converter can effectively realize coordinated power distribution and bus voltage stabilization of the microgrid. A mathematical model of grid-connected converter was established first in this paper, followed by a simulation analysis of the grid-connected converter under direct voltage control. In consideration of the fact that the ideal dynamic voltage response could not be realized by conventional control of the grid-connected converter, a seamless switching control strategy of inhibiting voltage fluctuation was proposed based on the voltage fluctuation analysis of the AC/DC converter, which improved the robust stability of the DC microgrid. The effectiveness and feasibility of the proposed control strategy were verified through the simulation results.
\end{abstract}

\section{Introduction}

As efficient, environmentally friendly and flexible power generation method, distributed generations (DGs) have exploited the advantages to the full in aspects of substituting traditional fossil energy resources, relieving environmental pollution and mitigating climate warming [1-2]. Microgrid has aroused extensive attention in the academic circles and industrial circles as an effective method of autonomously integrating DGs, energy storage unit and multi-type loads. A DC microgrid can realize both grid-connected operation but also isolated island operation. Under the grid-connected operation mode, the photovoltaic and energy-storage DC microgrid can contribute to reasonable application of photovoltaic power source and energy storage; The isolated island operation mode can solve the power supply problems for remote areas and places without power grid [3-4]. However, the safe and reliable operation of microgrid system is seriously threatened by various factors, e.g. fluctuation of DG power output and load power, and system low-inertia characteristic and complication of grid-connected converter structure due to the access of a large quantity of flexible power electronic equipment. No reactive power exists in any DC microgrid, so DC bus voltage is the only index used to measure the safe and stable operation of a DC microgrid.
The present researches on bus voltage of DC microgrid, from generation to multi-source coordination control, disturbance feedforward control algorithm to DC bus-side electric energy quality control, have become quite mature. Literature [5] proposed a power feedforward control method based on BTB converter, and $\mathrm{DC} / \mathrm{DC}$ and $\mathrm{DC} / \mathrm{AC}$ two-stage system; Literature [6] put forward introducing the load current feedforward method into the bus voltage control device of the DC microgrid. Most of the abovementioned researches have made improvements based on traditional proportionintegral double closed loop control, the control effect has high reliance on two parameters - proportional gain and integral time, and it is very sensitive to system parameters, so no ideal control effect can be achieved.

To solve the above problems, energy storage unit will be added on the traditional control basis. This method can make rapid response to operation mode switching of the grid-connected current converter and realize high stability of DC bus voltage in the operation mode switching process, and moreover, it has favorable dynamic performance.

\section{Topology of typical DC microgrid}

The topological structure of a typical DC microgrid is shown in Fig. 1.1, which mainly includes DG, energy storage device, DC load and related power electronic device.

*Corresponding author's e-mail: 283035393@qq.com 


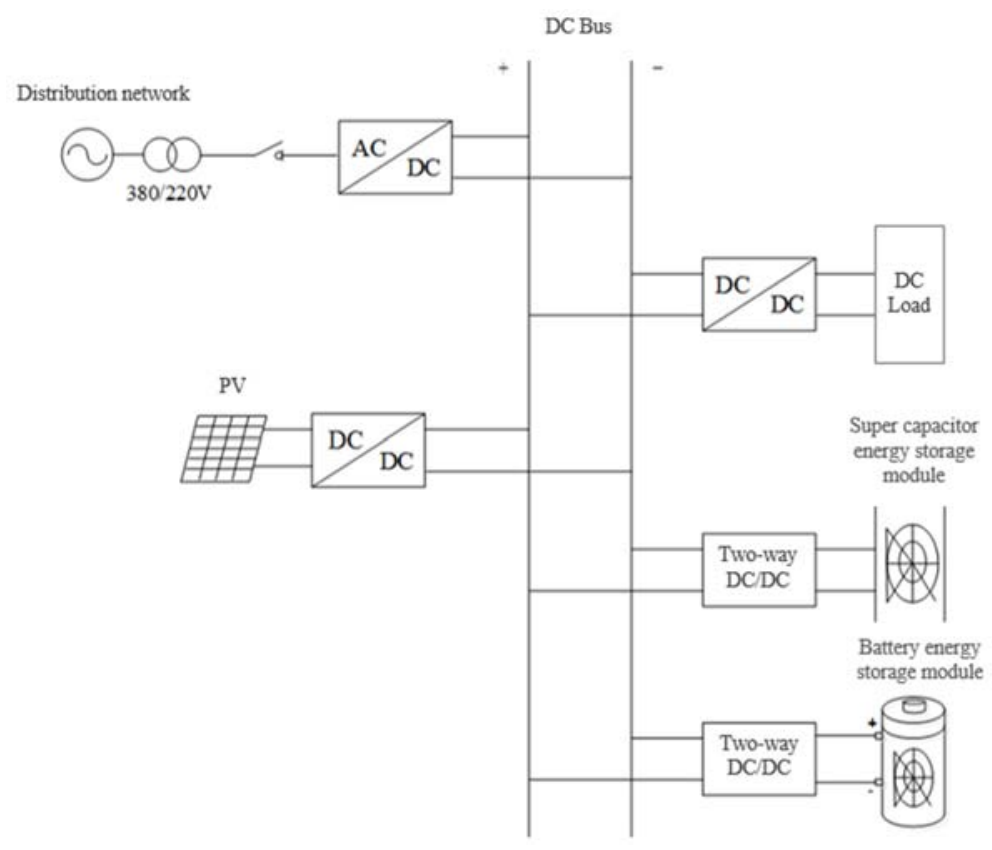

Fig. 1.1 Structural composition of DC microgrid

\section{Mathematical model of grid-connected current converter}

Grid-connected converter (GCC) is the two-way power flow interface between DC microgrid and large power grid. Under the grid-connected operation mode of the DC microgrid, the grid-connected current converter usually controls the stability of DC bus voltage; The gridconnected current converter will be locked upon the fault occurrence in the large power grid to disconnect the large power grid and microgrid, and in this way, the microgrid will not be influenced by the large power grid and isolated island operation is realized. The topological structure of the grid-connected current converter is shown in Fig. 2.1, where $e_{a}, e_{b}$ and $e_{c}$, and $i_{a}, i_{b}$ and $i_{c}$ are three-phase AC voltages and currents of the GCC, respectively; $u_{a}, u_{b}$ and $u_{c}$ are three-phase AC voltages of the power grid; $r$ and $L$ are connecting resistance and inductance of the converter, respectively; $i_{d c}$ denotes the current output by the current converter; $C$ is DC filtering capacitance; $u_{d c}$ represents DC voltage.

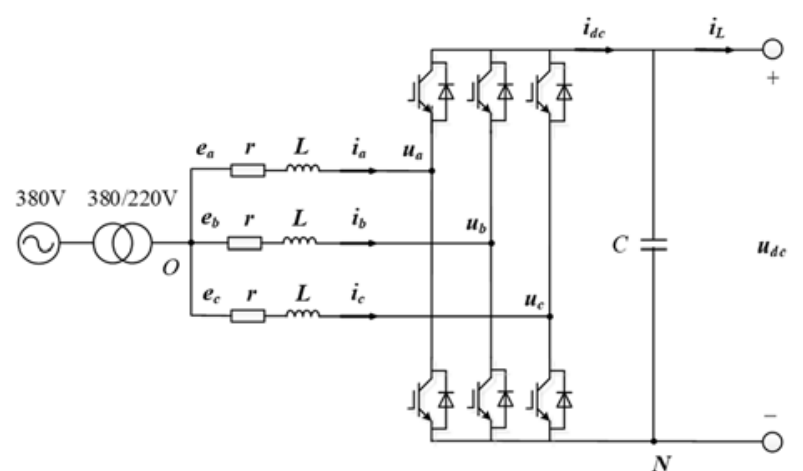

Fig. 2.1 Topological structure of grid-connected current converter

In the three-phase static coordinate system, the $\mathrm{AC}$ side of the grid-connected converter can be expressed according to Kirchhoff Voltage Law as below:

$$
\left[\begin{array}{l}
e_{a} \\
e_{b} \\
e_{c}
\end{array}\right]=\left[\begin{array}{l}
u_{a} \\
u_{b} \\
u_{c}
\end{array}\right]+L \frac{d}{d t}\left[\begin{array}{l}
i_{a} \\
i_{b} \\
i_{c}
\end{array}\right]+r\left[\begin{array}{l}
i_{a} \\
i_{b} \\
i_{c}
\end{array}\right]
$$

With the three-phase equilibrium condition of grid voltage, the mathematical model of GCC under the synchronous rotating coordinate system can be expressed as follow:

$$
\left[\begin{array}{l}
e_{d} \\
e_{q}
\end{array}\right]=\left[\begin{array}{l}
u_{d} \\
u_{q}
\end{array}\right]+L\left[\begin{array}{rr}
0 & -\omega \\
\omega & 0
\end{array}\right]\left[\begin{array}{l}
i_{d} \\
i_{q}
\end{array}\right]+L \frac{d}{d t}\left[\begin{array}{l}
i_{d} \\
i_{q}
\end{array}\right]+r\left[\begin{array}{l}
i_{d} \\
i_{q}
\end{array}\right]
$$

Where $e_{d}$ and $e_{q}$ are $d$ and $q$-axis components of three-phase AC grid voltage, respectively; $i_{d}$ and $i_{q}$ denote $d$ and $q$-axis components of three-phase AC voltage of the GCC, respectively; $u_{d}$ and $u_{q}$ express 
$d$ and $q$-axis components of three-phase AC voltage of the GCC, respectively; $\omega$ is angular frequency of grid voltage.

\section{Module control strategies for the DC microgrid}

The control modes for different unit modules in the DC microgrid are listed in Tab. 3.1. Under the gridconnected operation mode, the DC bus voltage is stabilized by the GCC, and both energy storage units assist in power balance via droop control; Under the isolated island operation mode, the supercapacitor energy storage unit with fast dynamic response is transformed into direct voltage control while droop control is still adopted by the energy storage battery. To take full advantages of DGs, the photovoltaic module operates under the Maximum Power Point Tracking (MPPT) mode during both grid-connected and isolated island operations.

\begin{tabular}{ccccc} 
Table 1. Operation mode of DC microgrid. \\
\hline Operation mode & $\begin{array}{c}\text { Control method for grid- } \\
\text { connected converter }\end{array}$ & $\begin{array}{c}\text { Control mode of } \\
\text { supercapacitor energy } \\
\text { storage module }\end{array}$ & $\begin{array}{c}\text { Control mode of } \\
\text { battery energy storage } \\
\text { module }\end{array}$ & $\begin{array}{c}\text { Control mode of } \\
\text { photovoltaic power } \\
\text { generation module }\end{array}$ \\
\hline $\begin{array}{c}\text { grid-connected } \\
\text { operation mode } \\
\text { isolated island } \\
\text { operation mode }\end{array}$ & direct voltage control & droop control & droop control & MPPT \\
locked & direct voltage control & Improved drop control & MPPT
\end{tabular}

\subsection{Control method of the grid-connected current converter}

It can be known from the above analysis that the gridconnected current converter has two operation modes, namely direct voltage control mode and locking mode. When the former is adopted, PI controller will be used for both inner current loop and outer voltage loop. It can be known that variables $d q$ are mutually coupling, the feedforward decoupling method should be used, and then:

$$
\left\{\begin{array}{l}
u_{d}=-\left(K_{i P}+\frac{K_{i I}}{s}\right)\left(i_{d}^{*}-i_{d}\right)+\omega L i_{q}+e_{d} \\
u_{q}=-\left(K_{i P}+\frac{K_{i I}}{s}\right)\left(i_{q}^{*}-i_{q}\right)-\omega L i_{d}+e_{q}
\end{array}\right.
$$

(a) Block diagram of d-axis control
Where $K_{i P}$ is proportionality coefficient and $K_{i I}$ is integral coefficient.

The following can be obtained through simultaneous equations (2-2) and (3-1):

$$
\left\{\begin{array}{l}
\left(K_{i P}+\frac{K_{i I}}{s}\right)\left(i_{d}^{*}-i_{d}\right)=(s L+r) i_{d} \\
\left(K_{i P}+\frac{K_{i I}}{s}\right)\left(i_{q}^{*}-i_{q}\right)=(s L+r) i_{q}
\end{array}\right.
$$

As seen in Eq. (3-2), $i_{d}$ and $i_{q}$ are decoupled, and the block diagram of the current loop control after addition of sampling delay and PWM small inertia link is shown in Fig. 3.1. $T_{s}$ is PWM switching period and the intermediate link is the result of joint action of sampling delay and PWM small inertia.

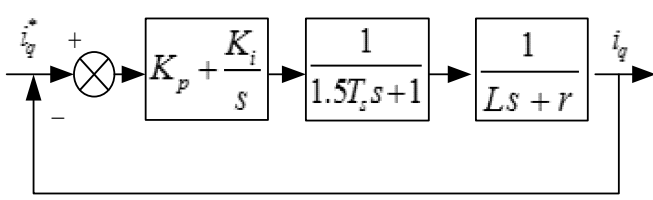

(b) Block diagram of q-axis control

Fig. 3.1 Block diagram of inner current loop

The block diagram of the grid-connected converter control under the direct voltage control mode is seen in Fig. 3.2. When voltage and current double closed loop control is adopted, the output of outer voltage loop is the reference current input of the inner current loop, and the inner current loop can effectively improve the dynamic response performance. 


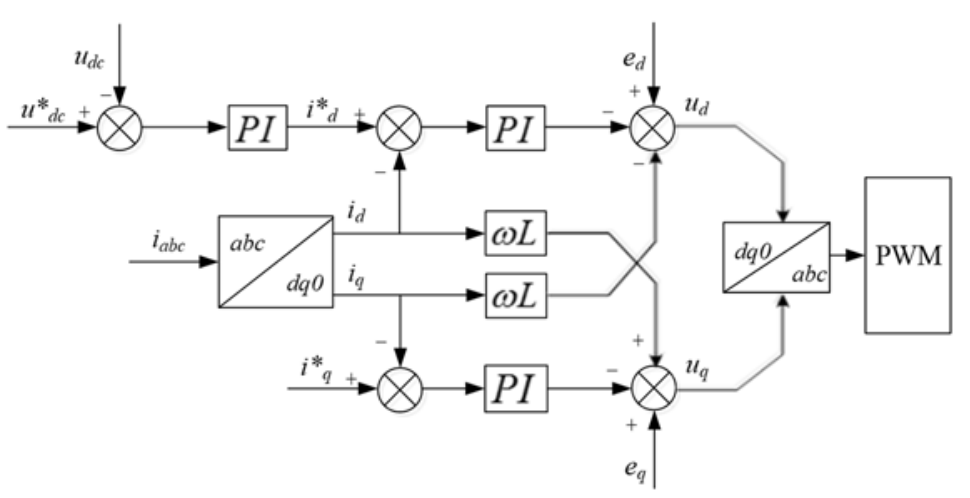

Fig. 3.2 Voltage control diagram of the grid-connected current converter

The energy storage unit in the established DC microgrid model has two energy storage modules: supercapacitor energy storage module and storage battery energy storage module. The control method of the energy storage unit is shown in Fig. 3.3.

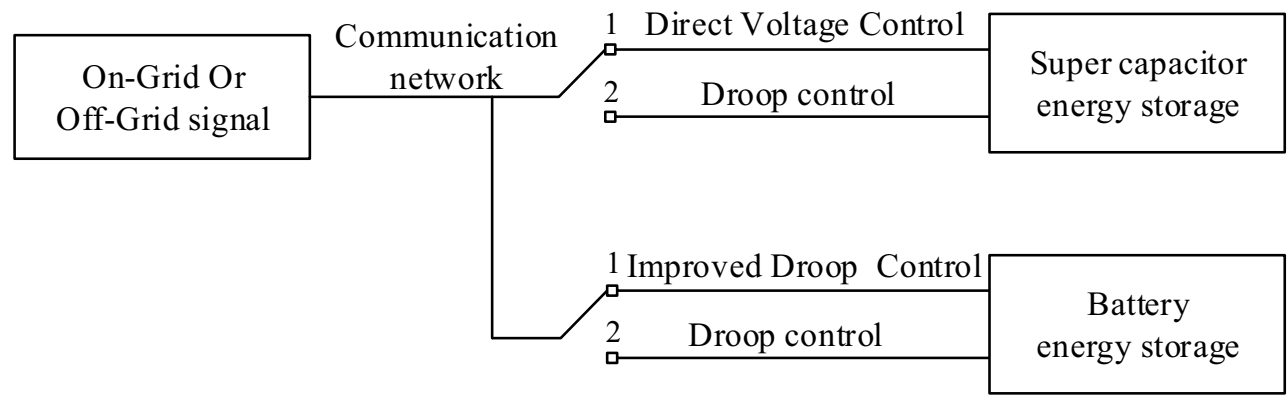

Fig. 3.3 Control method of energy storage unit

The upper controller conveys grid-connecting and off-grid signals to the energy storage unit via communication network. The upper and lower switches will select Channel 2 when the microgrid connected to the large power grid. During the direct voltage control of the GCC, both supercapacitor and storage battery assist in GCC charging and discharging through droop control in order to maintain the power balance; The upper and lower switches will choose Channel 1 when the DC microgrid is off the large power grid.

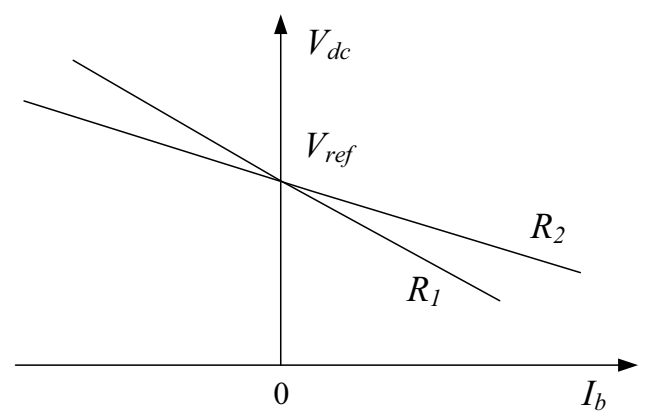

Fig. 3.4 Schematic diagram of droop control

The droop control has been extensively applied to $\mathrm{AC} / \mathrm{DC}$ microgrids, and the schematic diagram of droop control of the DC microgrid is shown in Fig. 3.4. The main idea of droop control is to keep the droop coefficients of converters different and implement reasonable distribution of the needed load power.

From the figure, the current and voltage output by each converter satisfy the following relation:

$$
V_{o}=V_{\text {ref }}-I_{o} R_{o}
$$

Where $V_{o}$ and $I_{o}$ are output voltage and output current, respectively; $V_{\text {ref }}$ is rated bus voltage; $R_{o}$ is droop coefficient, namely equivalent output impedance of current converter. $V_{d c}<V_{\text {ref }}$ means insufficient power in the system and $I_{b}>0$, namely the energy storage operates under discharging mode, and the power is output at the time; When $V_{d c}>V_{\text {ref }}$, surplus power exists in the system and $I_{b}<0$, namely the energy storage operates under charging mode and power is absorbed at the time. Therefore, when multiple converts have different droop coefficients and if $R_{1} \neq R_{2}$, the output power of the converter satisfies the relational expression $P R_{1}=P R_{2}$, and the power distribution among the converters can be determined by the same proportion according to their maximum output powers. 


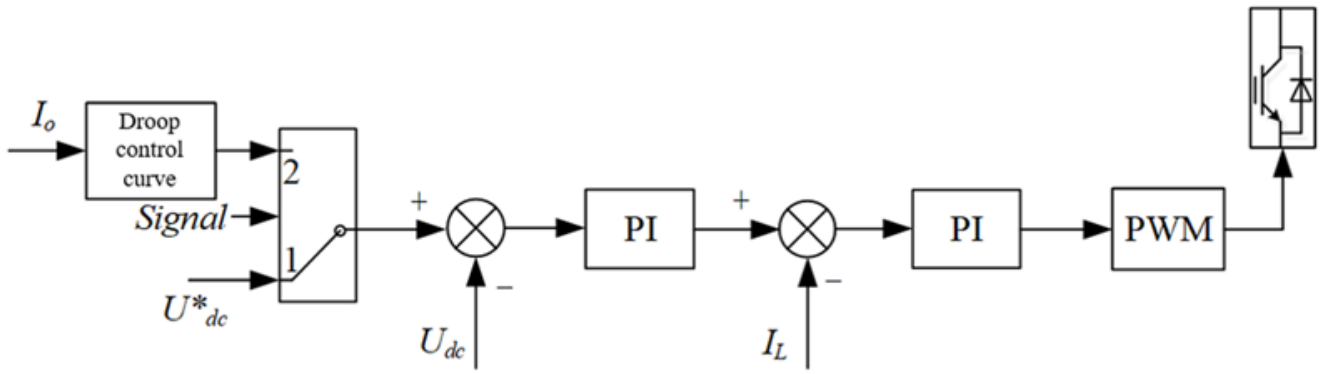

Fig. 3.5 Control diagram of supercapacitor energy storage module

The control of the supercapacitor energy storage module is shown in Fig. 3.5. The mode discrimination is realized according to grid-connecting/off-grid signals, where grid-connecting signal is expressed by 1 and offgrid signal by 0 . When Signal=1, Channel 2 will be selected by the switches; When Signal $=0$, Channel 1 will be selected.

\subsection{Seamless switching control strategy}

The switching between grid-connected operation and isolated island operation may result in voltage oscillation or sharp decrease. Smooth and fast switching process is an important index used to measure whether a microgrid control strategy is effective. According to the mechanism analysis of bus voltage oscillation at switching time, the power vacancy at GCC port should be immediately compensated in order to shorten the dynamic response time. The effect will be unideal if only energy-storage outer voltage loop control is used or the improved direct voltage control method is used. Therefore, a supercapacitor energy storage module is considered in this paper, which can instantaneously enlarge the output power at switching time. The supercapacitor has faster dynamic response than the storage battery, and moreover, the direct voltage control method is used so that the two energy storage units can cooperate with each other to better improve the dynamic performance of the system.

If the load power is unchanged before and after switching, the photovoltaic power will be output according to maximum power, and after switching, GCC power, energy storage battery port and energy-storage supercapacitor port will satisfy the following relation:

$$
P_{G C C}+P_{\text {battery }}+P_{C}=P_{\text {battery }}^{\prime}+P_{C}^{\prime}
$$

Using the direct voltage control mode after supercapacitor switching will fail to compensate for the power vacancy at the original GCC port. Therefore, compensating through droop control of the energy storage battery is a relatively reasonable scheme, and the improved droop control diagram is shown in Fig. 3.6.

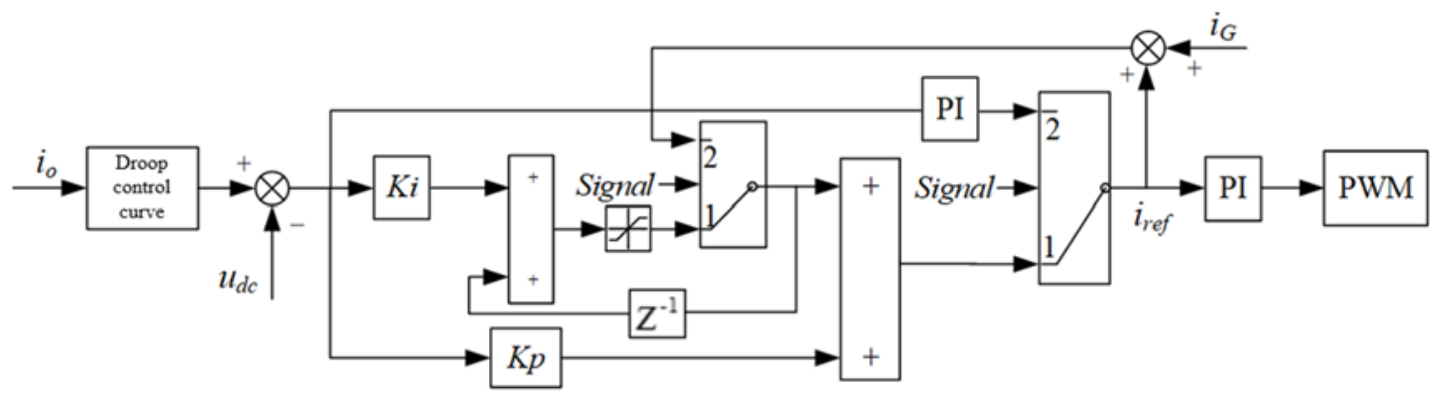

Fig. 3.6. The improved droop control diagram

When the DC microgrid is under grid-connected operation, the grid-connecting signal will select Channel 2 , the reference voltage at the time is calculated through the droop curve, and PWM modulation is obtained through voltage and current double closed loop output. Meanwhile, the sum of the reference current, which is under droop control, and GCC DC current $i_{G}$ is continuously tracked, and Channel 1 only calculates data but not outputs the result. When a grid-side fault forces the DC microgrid to switch to isolated island operation, the off-grid signal will choose Channel 1, and the instantaneous output of the outer voltage loop increases the GCC current before switching, and then:

$$
i_{\text {ref }}^{\prime}=i_{r e f}+i_{G}
$$

In other words, the power satisfies the following equation:

$$
P_{\text {battery }}^{\prime}=P_{\text {battery }}+P_{G C C}
$$

Eq. (3-5) indicates that the improved droop control can compensate for the power vacancy at GCC port very well. 


\section{Simulation analysis}

A DC microgrid simulation model was established in MATLAB/Simulink simulation software. The model was composed of photovoltaic power generation module, Boost circuit used for power control, energy storage unit, load, etc.

\subsection{Simulation analysis of DC microgrid control strategy}

Under the grid-connected operation mode, direct voltage control is adopted for the grid-connected current converter, the rated voltage of grid-side output line is 220

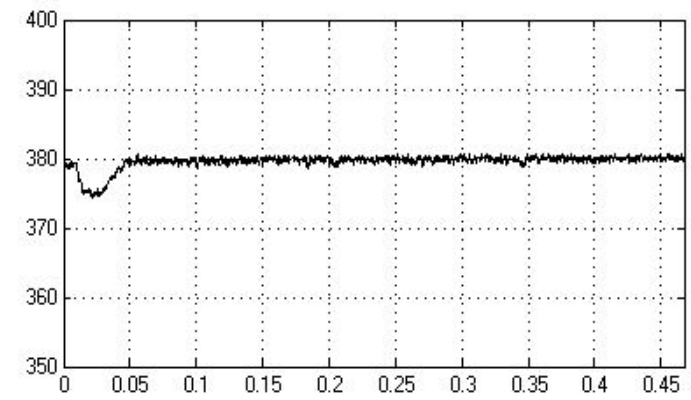

Time offset: 0

(a) DC bus voltage waveform
$\mathrm{V}$ and the port power does not exceed 5,000 W. Assume that external environmental conditions are ideal in the operation process, the temperature is $25^{\circ} \mathrm{C}$, light irradiance is $1,000 \mathrm{~W} / \mathrm{m} 2$, and the theoretical maximum output power of the photovoltaic module is about 5,000 $\mathrm{W}$. The rated output voltage and rated power at the load end are $200 \mathrm{~V}$ and $6,667 \mathrm{~W}$, respectively. The simulation time is set as $1 \mathrm{~s}$, the DC microgrid is under gridconnected operation within $0-0.5 \mathrm{~s}$, the DC microgrid system is disconnected from the large power grid at $\mathrm{t}=0.5$ $\mathrm{s}$ and transmits isolated island operation signal to the current converter. The DC microgrid is under isolated island operation within $0.5-1 \mathrm{~s}$.

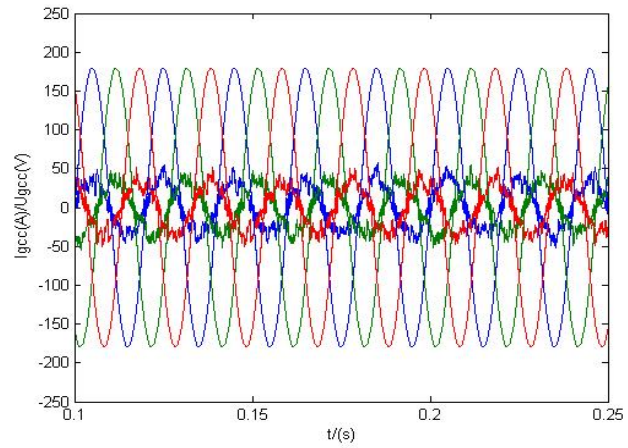

(b) GCC output AC voltage and current waveforms

Fig. 4.1 Simulation waveform under grid-connected mode

Fig. 4.1 (a) shows the waveform of DC bus voltage under the grid-connected mode. The DC microgrid is under grid-connected operation at $\mathrm{t}=0 \mathrm{~s}$. After about 0.05 $\mathrm{s}$ of dynamic response, the DC voltage is stabilized at $380 \mathrm{~V}$. It can be seen that the DC microgrid system is kept stable during the grid-connected operation period $(0-0.5 \mathrm{~s})$, so the direct voltage control strategy of the GCC is effective. Fig. 4.1 (b) displays the waveforms of

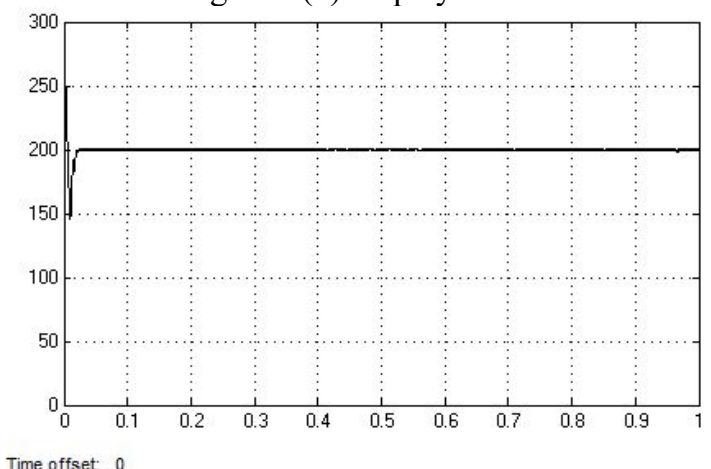

Fig. 4.2 DC load voltage waveform

Fig. 4.2 and Fig. 4.3 show DC load voltage and output power at photovoltaic port, respectively. It can be seen from Fig. 4.2 that during the whole DC microgrid operation period, the load-end voltage is of favorable stability thanks to the direct voltage control of buck circuit. The simulation model built in this paper can realize uninterrupted power supply to important loads in the DC microgrid, and furthermore, the load voltage is three-phase AC voltage and current output by GCC, which operates steadily under the grid-connected mode. It can be seen from the figure that the three-phase voltages and three-phase currents are in the same phase, and the output power factor reaches 1 because of GCC control. Besides, the waveforms manifest that the output current ripple is large when only inductance filter is used.

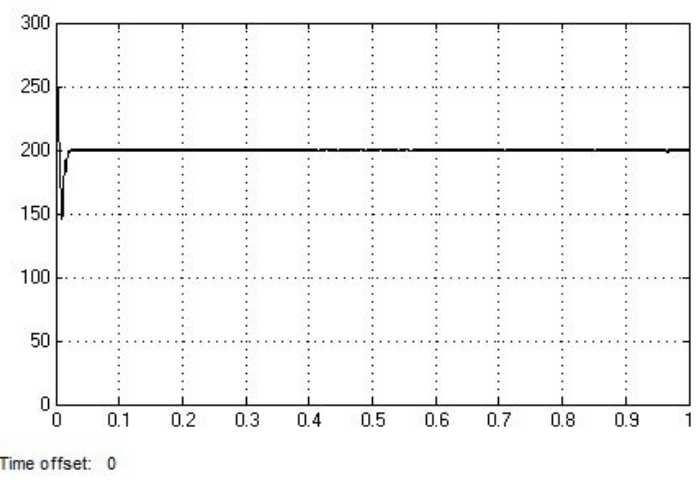

Fig. 4.3 Output power waveform of photovoltaic port

almost not disturbed during the switching process of operation mode, thus avoiding the possible load loss due to too large voltage oscillation and improving the robust stability of the DC microgrid. 


\subsection{Simulation analysis of operation mode switching control strategy for the DC microgrid}

The proposed grid-connected converter control strategy is verified through simulation, and the operation mode

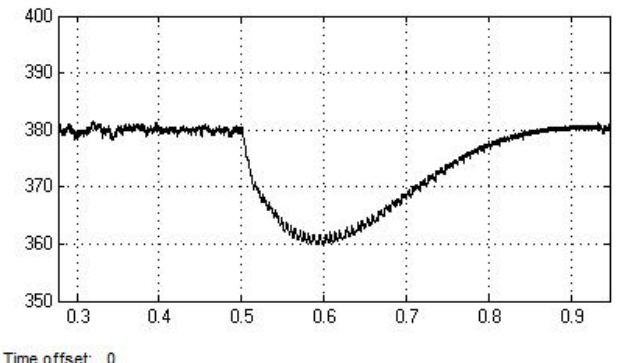

(a) Traditional control strategy switching time is still set as $0.5 \mathrm{~s}$. In the traditional control mode, the energy storage unit only contains the supercapacitor. Storage batteries are added to the operation mode switching strategy to improve the droop control-assisted switching method.

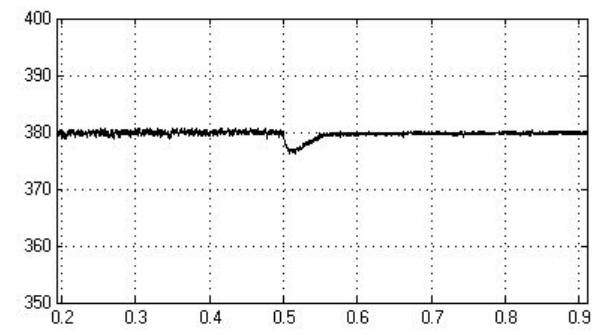

Time offset: 0

Fig. 4.4 DC bus voltage simulation waveform

The DC bus voltage simulation waveforms in the DC microgrid under the traditional control strategy are presented in Fig. 4.4 (a). It can be seen that during the off-grid process of the DC microgrid, the DC bus voltage reaches as low as about $360 \mathrm{~V}$, reducing by about $20 \mathrm{~V}$, which is about $5.3 \%$ of the rated voltage. Moreover, the response time spent in recovering the rated voltage from the trough $360 \mathrm{~V}$ is about $0.4 \mathrm{~s}$. Fig. 4.4 (b) is the DC bus voltage simulation waveform in the DC microgrid when the operation mode switching strategy is added. As shown in the figure, the maximum decrease amplitude of DC bus voltage is $5 \mathrm{~V}$, being about $1.3 \%$ of the rated voltage, and the duration of the transient process is about $0.05 \mathrm{~s}$. In comparison with the direct switching situation of the DC microgrid under the traditional control mode, the stability and reliability of the DC microgrid are considerably improved when the operation mode switching strategy is introduced.

\section{Conclusion}

A simulation model of the DC microgrid system in which photovoltaic module served as DG was established in this paper to simulate two steady operating conditions: grid-connected and off-grid operations. The simulation results manifested the effectiveness of the proposed control strategy. To solve the large fluctuation problem of DC bus voltage in the operation mode switching process of the DC microgrid, a supercapacitor module-based bus voltage control strategy was put forward. The simulation results show that, compared with the traditional control strategy, the proposed control strategy can maintain the voltage stability very well and effectively improve robust stability of the DC microgrid with excellent dynamic performance.

\section{References}

1. Wang Chengshan, Wu Zhen, Li Peng. (2014) Research on key technologies of microgrid[J]. Transactions of China Electrotechnical Society , 29(2): 1-12.

2. Pinson P, Nielsen H A, Madsen H, et al. (2013) Skill forecasting from ensemble predictions of wind power[J]. Applied Energy, 86(7): 1326-1334.

3. Li Xialin, Guo Li, Huang Di, et al. ( 2019 ) Research review on operation and control of DC distribution networks $[\mathrm{J}]$. Proceedings of the CSEE,45(10): 3039-3049.

4. Guo Li, Feng Yibin, Li Xialin, et al. ( 2016 ) Stability analysis and research of active damping method for DC microgrids [J]. Proceedings of the CSEE , 36(4): 927-936.

5. Vijayakumari A, Devarajan A T, Devarajan N. ( 2015 ) Decoupled Control of Grid Connected Inverter with Dynamic Online Grid Impedance Measurements for Micro Grid Applications[J]. International Journal of Electrical Power \& Energy Systems , 68: 1-14.

6. Wang J, Yan J D, Jiang L, et al. (2015) Delaydependent Stability of Single-loop Controlled Grid connected Inverters with LCL Filters[J]. IEEE Transactions on Power Electronics, 31(1): 743-757. 